\title{
Demanda de Internet: el sector mayorista de telecomunicaciones colombiano
}

Recibido: 31 de marzo de 2016 - Aceptado: 20 de octubre de 2016

Doi: dx.doi.org/10.12804/revistas.urosario.edu.co/economia/a.5624

\author{
John Jairo García Rendón* \\ Juan Pablo Posada Aparicio ${ }^{\dagger}$
}

\section{Resumen}

A raíz de la necesidad de conocer las diferentes variables que in- ciden en los servicios de transporte de datos, de escasez de artículos académicos que describan el comportamiento de la demanda en el sector mayorista y tomando como referencia los trabajos realizados, por Madden y Coble-Neal (2004) y Karacuka, Haucap y Heimeshoff (2011), se presenta, tanto un modelo de demanda, como un modelo de formación de precios, estimados por medio de técnicas de panel de datos dinámicos a nivel de firmas, con el objetivo de comprender el comportamiento de estas en el mercado colombiano; y cuyos resultados fundamentales son una elasticidad precio de la demanda de $-0,2768$, una elasticidad inversa de la demanda de $-0,0288$ y una elasticidad ingreso de 0,038 , para las firmas proveedoras del servicio de Internet a usuarios finales.

Palabras clave : desempeño de las firmas, precios, innovación, Internet. Clasificación JEL: C59, D22, L10

* Economista, Phd en Economía. Profesor Departamento de Economía, Universidad EAFIT, Colombia. AA3300 Medellín (Colombia). Teléfono: (+57 4) 261 9549, Fax: (+57 4) 266 4884. Correo electrónico: jgarcia@eafit.edu.co

+ Economista. Auxiliar de Investigación, Departamento de Economía, Universidad EAFIT, Colombia. Correo electrónico: jposad28@eafit.edu.co

Para citar este artículo: García, J.J., \& Posada, J.P. (2016): Demanda de Internet: el sector mayorista de telecomunicaciones colombiano. Revista Economía del Rosario, 19(2), 175-199. Doi: dx.doi.org/10.12804/revistas.urosario.edu.co/economia/a.5624 


\title{
Internet Demand: The Colombian Wholesale Telecommunications Sector
}

\begin{abstract}
Following the need to know the different variables that affect the data transport services, the shortage of academic papers that describe the behavior of the demand in the telecommunications wholesalers market and taking as a reference the work done by Madden y Coble-Neal (2004) and Karacuka, Haucap y Heimeshoff (2011), it's presented both a demand model, as well as a price formation model, estimated trough firms-level panel data techniques, aiming to understand the behavior of those firms in the Colombian market and which main results are a demands price elasticity of -0.2768 , an inverse price elasticity of -0.0288 and finally an income elasticity of 0.038 , for the firms providing the services to final client.
\end{abstract}

Keywords: firm performance, prices, innovation, Internet.

JEL Classification: C59, D22, L10

\section{Demanda de Internet: O Setor Maiorista de Telecomunicações Colombiano}

\author{
Resumo
}

Dada a necessidade de conhecer as diferentes variáveis que incidem nos serviços de transporte de dados, a escassez de artigos acadêmicos que descrevam o comportamento da demanda no setor maiorista e tomando como referência os trabalhos realizados, por Madden \& Coble-Neal (2004) e Karacuka, Haucap \& Heimeshoff (2011), se apresenta, tanto um modelo de demanda, quanto um modelo de formação de preços, estimados através de técnicas de painel de dados dinâmicos no nível de empresas, com o objetivo de compreender o comportamento destas no mercado Colombiano e cujos resultados fundamentais são uma elasticidade preço da demanda de -0.2768 , uma elasticidade inversa da demanda de -0.0288 e uma elasticidade ingresso de 0.038 , para as empresas provedoras do serviço de internet a usuários finais.

Palavras-chave: desempenho das empresas, preços, inovação, Internet.

Clasificación JEL: C59, D22, L10

\section{Introducción}

El sector de las telecomunicaciones en Colombia es, como resultado del aumento de la demanda de servicios, uno de los que presenta un mayor crecimiento en el país, triplicando en solo tres años la oferta del mercado y con expectativas de triplicarla nuevamente para 2018 (Telegeography, 2015; Internexa, 2012). Es un mercado con crecimientos exponenciales, con grandes oportunidades, pero que exige un monitoreo y revisión constante del portafo- lio ofrecido (Cusano, 2014; pwc, 2015), este crecimiento del sector trae retos muy importantes, ya que este tipo de comportamientos de la oferta resulta en una caída igual de 
dramática en los precios de los productos (Jankowski, Covello, Ritchie y Costa, 2014). Solo en el último año los precios por mega bits por segundo (Mbps) en el servicio de internet IP (Internet Protocol) han caído un 50 \% y la entrada de cables submarinos de distintas firmas es una amenaza constante para la estabilidad de los precios en el mercado (Internexa, 2014).

En el sector de mayoristas en el mercado colombiano destacan empresas como Level 3, C\&W, Media Commerce, Claro, Une, Telefónica e Internexa (Internexa, 2014), las cuales se especializan en proveer servicios a las empresas que le proporcionan servicios a los usuarios finales (con la excepción de Claro, Une y Telefónica, que tienen sus propios servicios). Son estas empre- sas las primeras en la cadena de servicios, que dan como resultado el servicio de transporte de datos que el consumidor final recibe, es por esto que los cambios en la demanda de los servicios a nivel de los mayoristas repercute en los precios que percibe el consumidor final; de ahí la importancia de este estudio, ya que su comprensión y modelación, poco estudiado puede dar luces a futuras investigaciones sobre los sectores minoristas.

En la tabla 1 se realiza una comparación para algunos mercados importantes con el objetivo de informar al lector el tamaño del mercado colombiano. Colombia se ubica en un nivel intermedio en América Latina, por debajo de Brasil, Argentina y Chile.

Tabla 1. Comparacion de mercados mayoristas internacionales

\begin{tabular}{lcc} 
& Gbps (2015) & Crecimiento en \% (2015) \\
\hline Colombia & 1784 & 49 \\
Ecuador & 295 & 49 \\
Venezuela & 733 & 49 \\
Perú & 1605 & 38 \\
Chile & 2775 & 34 \\
Brasil & 10247 & 43 \\
Argentina & 3638 & 44 \\
América Latina & 25066 & 42 \\
EE.UU. & 87890 & 41 \\
España & 8871 & 26 \\
Francia & 54156 & 37 \\
Alemania & 75100 & 37 \\
Europa & 155418 & 37 \\
\hline
\end{tabular}

Fuente: elaboración propia a partir de Telegeography (2015). 
Así el objetivo general que tiene esta investigación, es analizar cuáles son los principales determinantes que afectan la formación del precio de la industria mayorista de telecomunicaciones en Colombia y, a su vez, los principales determinantes de la demanda en este sector. Para lograr esta meta, se apoya en los siguientes objetivos específicos: i) estimar las elasticidades precio e ingreso de la demanda, controlando por otras variables que determinan el desempeño del sector, ii) estimar la función de precios para este mercado, iii) realizar un análisis de las principales variables que afectan el funcionamiento del mercado, objeto de estudio.

Es de vital importancia comprender el papel que cumple el proceso determinador de precios en las firmas mayoristas en telecomunicaciones, para así contar con una visión holística del mercado y poder añadir a los estudios, investigaciones y regulaciones en los mercados minoristas, los escenarios propios de un mercado comúnmente excluido.

Mediante la utilización del método de Blundell y Bond, con el fin de obtener estimaciones eficientes e insesgadas a nivel de firmas, al igual que Karacuka et al. (2011), se estiman tanto una función de demanda, como una función de formación de precios en el mercado mayorista para el periodo comprendido entre enero de 2008 y diciembre de 2015. Dichas funciones son utilizadas como instrumento para obtener las elasticidades: precio de la demanda, ingreso (para las firmas que proporcionan el servicio a usuarios finales) e inversa de la demanda.

Vale la pena resaltar el valor que tiene el estudio como proveedor de información del mercado colombiano, ya que permite no solo un análisis histórico de la evolución de las tarifas y los anchos de banda, sino una presentación de los principales determinantes de la demanda. Este tipo de análisis puede ser utilizado como fuente de información primaria para los entes reguladores, con el objetivo de formular mejores políticas que incentiven a los actores para que haya una mejor prestación de servicio al usuario final (OECD, 2014), ya que en el país existe, además de un vacío institucional que repercute en una regulación insipiente en el sector (OECD, 2014), un vacío de información esencial sobre las elasticidades en este sector.

Igual que la mayoría de mercados estudiados a nivel mundial (EE.UU., Turquía, Austria, Australia, Israel y España), Colombia presenta una elasticidad precio de la demanda inelástica, con un coeficiente de $-0,27$, siendo una de las menores. Además desde la elasticidad ingreso de la demanda del servicio de Internet se comporta como un bien normal necesario.

Este artículo se estructura de la siguiente manera: la sección uno, hace un recuento de la revisión de literatura, además de comparar los resultados obtenidos con los de otras investigaciones relevantes. La sección dos presenta 
el enfoque empírico. En la sección tres se presenta la metodología utilizada y en las secciones cuatro y cinco se presentan los resultados y las conclusiones.

\section{Revisión de literatura}

La importancia de esta investigación surge como resultado de la necesidad de comprender y modelar el comportamiento de los precios en la industria mayorista de Internet, la cual, no habiendo sido explorada a profundidad, representa una oportunidad para la generación de conocimiento. Se trata de un mercado bastante dinámico, que refleja el comportamiento de la economía global hoy en día, altamente interconectado y de una evolución veloz (Cusano, 2014; Jankowski et al., 2014).

A raíz de la no existencia de estudios para mercados mayoristas, se hace uso de las investigaciones existentes para mercados cuyo consumidor es el usuario final del servicio (telefonía fija, telefonía móvil y servicios de Internet domiciliarios), especialmente las de Karacuka et al. (2011) y Madden y CobleNeal (2004). Por lo tanto, se realiza una revisión de los estudios que describen y permiten hacer uso de técnicas de modelación o soluciones a problemas específicos de investigaciones en los mercados de telecomunicaciones, que han sido objeto de estudio en otros países.

Nos concentramos en la revisión de los resultados econométricos obtenidos, en particular de las elasticidades precio de la demanda. La tabla 2 muestra los principales estudios realizados a nivel mundial, donde, si bien una de las industrias más estudiadas corresponde a la de Estados Unidos (Grzybowski, 2008; Rodini, Ward y Woroch, 2003; Hausman, 1999; Parker y Röller, 1997; Houthakker y Taylor, 1970), también se encuentran estudios para Turquía (Karacuka et al., 2011); para Israel (Tishler, Ventura y Watters, 2001) y para Australia (UK Competition Commission, 2003). Además de otras investigaciones como las de Madden y Coble-Neal (2004) y Ahn y Lee (1999), en las que el objeto de estudio ha sido un grupo bastante amplio de países.

Como puede observarse en la tabla 2, la elasticidad precio de la demanda puede variar dependiendo del área geográfica y del tipo de mercado, es decir, si es telefonía móvil o fija. Por ejemplo, para la industria de telefonía móvil prepago en Turquía, Karacuka et al. (2011) encuentran una elasticidad de $-0,33$, la cual corresponde a una de las elasticidades más bajas, seguida de la industria de telefonía móvil para Estados Unidos reportada en el trabajo de Grzybowski (2008) con -0,2, no obstante, en este estudio también encuentran elasticidades de hasta $-0,9$. 
Tabla 2. Comparación de elasticidades

\begin{tabular}{lcl}
\hline \multicolumn{1}{c}{ Autor y área geográfica del estudio } & Elasticidad & \multicolumn{1}{c}{ Mercado } \\
\hline Karacuka et al. (2011) - Turquía & $-0,72$ & Móvil Postpago \\
Karacuka et al. (2011) - Turquía & $-0,33$ & Móvil Prepago \\
Grzybowski (2008) - EE.UU. & Entre -0,2 y -0,9 & Móvil \\
Dewenter y Haucap (2008) - Austria & $-0,67$ & Móvil \\
Madden y Coble-Neal (2004) - 58 paises & $-1,15$ & Móvil \\
Rodini et al. (2003) - EE.UU. & $-0,43$ & Telefonía fija \\
UK Competition Commission (2003) - Australia & $-0,8$ & Móvil \\
Tishler et al. (2001) - Israel & $-0,8$ & Móvil \\
Hausman (1999) - EE.UU. & $-0,51$ & Móvil \\
Ahn y Lee (1999) - 64 paises & $-0,36$ & Móvil \\
Muñoz y Amaral (1996) - España & $-0,57$ & Móvil internacional \\
Muñoz (1996) & -0.13 & Móvil nacional \\
Parker y Röller (1997) - EE.UU. & $-2,5$ & Móvil \\
Houthakker y Taylor (1970) - EE.UU. & $-0,45$ & Múltiples \\
\hline
\end{tabular}

Fuente: elaboración propia a partir de Karacuka et al. (2011); Grzybowski (2008); Dewenter y Haucap (2008); Madden y Coble-Neal (2004); Rodini et al. (2003); UK Competition Commission (2003); Tishler et al. (2001); Hausman (1999); Ahn y Lee (1999); Muñoz y Amaral (1996); Muñoz (1996); Parker y R"oller (1997); Houthakker y Taylor (1970).

De la revisión de la literatura, los únicos dos estudios que obtienen una elasticidad precio de la demanda elástica, es decir superior a 1, son los trabajos realizados por Madden y Coble-Neal (2004) y el de Parker y Röller (1997) con elasticidades de $-1,15$ y -2,5 respectivamente, los demás estudios arrojan una elasticidad inelástica. Un aspecto que puede influir en las diferencias de los resultados obtenidos puede estar explicado por la técnica utilizada para la estimación, ya que en la medida en que se utilizan enfoques más acertados, como, por ejemplo, modelos dinámicos, los resultados pueden ser insesgados y eficientes y, por lo tanto, más confiables.

El estudio realizado por Karacuka et al. (2011) difiere de los resultados obtenidos respecto a las elasticidades de la investigación de Hausman (1999), quien al utilizar datos agregados de 30 mercados correspondientes a diferentes Estados de Estados Unidos, encuentra una elasticidad precio de la demanda para servicios de telefonía móvil de -0,51, pero es, a la vez, bastante cercano al resultado obtenido por Ahn y Lee (1999) quienes, usando una técnica de panel de datos para 64 países, encuentran una elasticidad de $-0,36$ para el mismo servicio. 
En el análisis de la literatura respecto a la metodología utilizada para la estimación, resalta la investigación realizada por Karacuka et al. (2011), en la que, con el fin de estructurar un modelo casi ideal de demanda, se hace uso de información a nivel de firmas y se fundamentan en la metodología propuesta por Houthakker y Taylor (1970), quienes estiman una función de demanda con sus respectivas elasticidades precio. También vale la pena resaltar el estudio de Dewenter y Haucap (2008), quienes utilizan un modelo dinámico para hacer las estimaciones. Así, la metodología utilizada por estos estudios se convierte en un referente para la investigación planteada en esta investigación, con algunas variaciones, dadas las diferencias presentadas en los mercados y acorde a la restricción de información disponible para la industria mayorista de Internet en Colombia.

\section{Enfoque empírico}

El mercado de telecomunicaciones ha sufrido en los últimos años, como resultado de la naturaleza basada en tecnología de su negocio, una caída muy pronunciada en el precio de sus productos y servicios. Esto ha forzado al mercado a ser extremadamente dinámico, al punto de presentarse fusiones y adquisiciones al interior del sector, como algo casi rutinario (Gruber, 1999).

Se trata de un mercado donde hay que practicar un doble juego: de mantener la compañía hoy y prepararla para el futuro, al mismo tiempo (Philipp G. y Steinhaüser, 2015). La innovación es una variable clave para sobrevivir, y las empresas con ánimo de subsistir en el tiempo están dispuestas, como se demostrará más adelante, a invertir grandes cantidades de dinero para este propósito, por esto, y con la finalidad de dar claridad sobre las variables utilizadas en la modelación, se presenta en la tabla 3 una descripción de estas.

Como se puede apreciar, tanto en la figura 1, como en la tabla 4, el precio promedio en el servicio de Internet Protocol (IP) ha sufrido una caída bastante pronunciada desde enero de 2008, pasando de un Mbps promedio de U\$D 247,5, a un nivel muy inferior en diciembre de 2015, ubicándose en el orden de los U\$D 20,3 por Mbps, con una alta variabilidad al interior de un mismo cliente. De ahí parte de la explicación, además de las variables presentadas a continuación, a la evolución tecnológica, siendo esta un factor endógeno del mercado, así como la entrada de importantes competidores, sobre todo a partir de 2008 con la licitación pública de Vive Digital, la cual dio como ganador a Azteca Comunicaciones y lo posicionó como un importante agente en el mercado mayorista de telecomunicaciones (OECD, 2014). 
La entrada de este nuevo competidor, entre otros aspectos, se ve reflejada en la caída abrupta que sufrieron los precios entre 2008 y 2009 y es además un determinante importante a la hora de analizar el mercado. Azteca Comunicaciones posee una capacidad inigualable en el mercado colombiano, llegando a algunos de los municipios más alejados del país, 472 en total (Azteca-Comunicaciones, 1999), y es esta característica la que le ha permitido posicionarse rápidamente como uno de los principales actores del mercado.

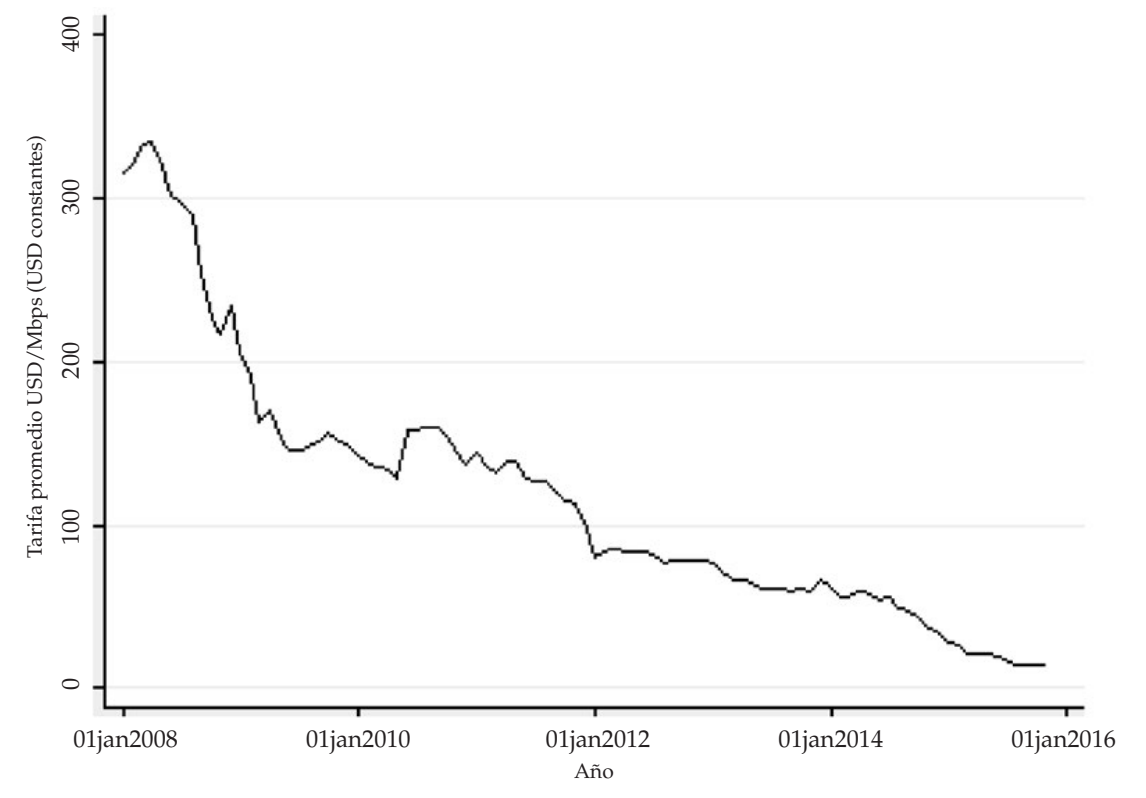

Figura 1. Tarifa promedio, servicio IP

Fuente: elaboración propia a partir de Internexa (2015).

Por otro lado, las cantidades en el mercado reflejan la caída abrupta de los precios descrita anteriormente. El crecimiento del ancho de banda sigue una tendencia cuasi exponencial, descrita por la ley de Moore (Schaller, 1997), pero exacerbada por el dinamismo comercial del mercado en los últimos años. La entrada de cables submarinos ha sido, cada vez, más común y las expectativas apuntan a que Colombia, dado su ingreso a la OCDE, se asemeje cada vez más a los mercados mayoristas de estos países, donde es normal que clientes no pertenecientes al segmento de los mayoristas se auto sirvan, gracias a las facilidades de ancho de banda ofrecidas por el mercado, ya sea en forma de fibra oscura o mediante la implementación de red propia (oecd, 2014; Internexa, 2014). 
La figura 2 permite apreciar, no solo el comportamiento exponencial, anteriormente descrito, sino también la correlación existente entre la evolución del IP ofrecido por Internexa y el ancho de banda total colocado en el mercado colombiano, presentando un Coeficiente de Correlación de Pearson de 0,962 (Coletti, 2010), lo cual permite asumir a Internexa como un agente representativo del mercado, detalle para nada despreciable, ya que esto significa que los resultados obtenidos mediante el análisis cuantitativo se pueden hacer extensivos al mercado y no hacen parte exclusiva del análisis de la firma. Con lo anterior, se pretende que el análisis aquí constatado, al igual que el propuesto por Karacuka et al. (2011), construya un modelo que permita hacer un análisis inductivo para generar conclusiones a nivel de mercado, estudiando la firma como agente económico fundamental.

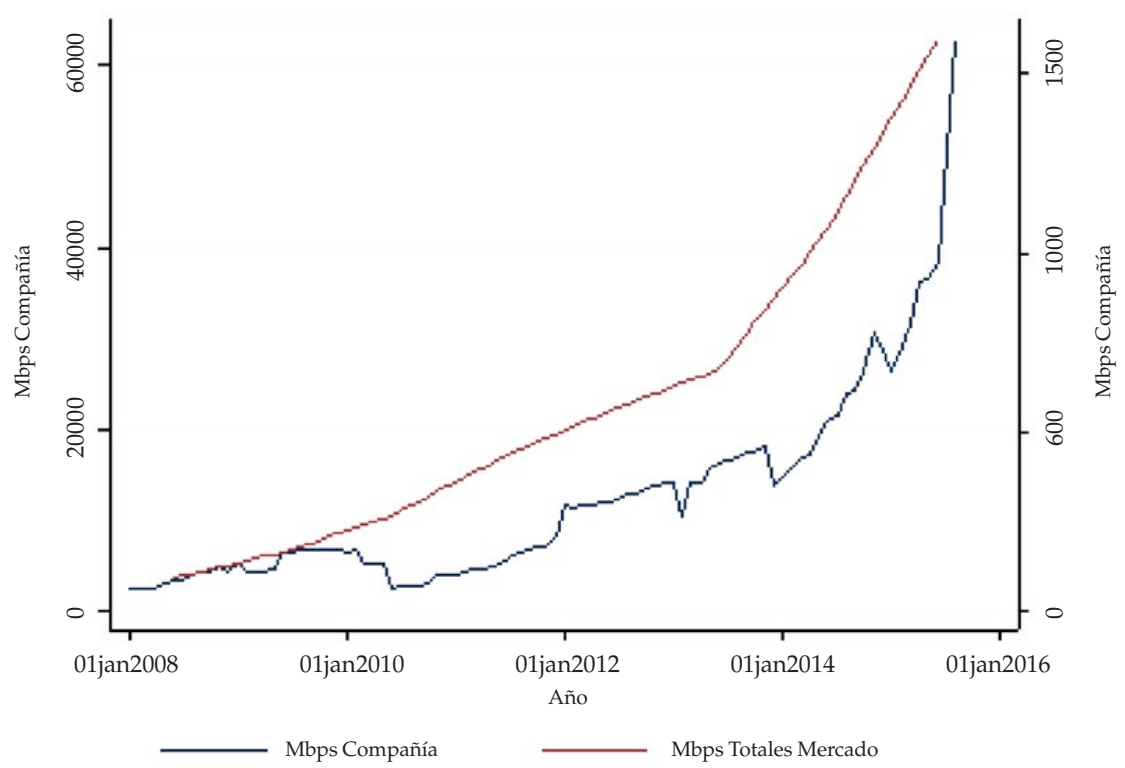

Figura 2. Mbps en la industria

Fuente: elaboracion propia a partir de Internexa (2015); Telegeography (2015).

Dado lo anteriormente expuesto, se torna interesante observar las capacidades, tal y como se muestran en la tabla 4 , ya que se puede ver que estas van desde un Mbps hasta poco más de tres gigas y media, pero esta gran variedad es aun mayor cuando se analiza la variación entre clientes. Se puede ver una gran dispersión, con un máximo de $6155 \mathrm{Mbps}$, lo que deja clara la gran heterogeneidad en los consumidores, también se aprecian unas variaciones 
enormes, al interior de un mismo cliente, que muestran el gran dinamismo de la industria ${ }^{1}$.

Tabla 3. Definición de Variables

\begin{tabular}{|c|c|c|c|}
\hline Variable & Descripción & Periodicidad & Fuente \\
\hline Tarifa & $\begin{array}{l}\text { Pago mensual que un cliente desem- } \\
\text { bolsa por su servicio de IP. Esta se } \\
\text { presenta en usd por Mbps. }\end{array}$ & Mensual & ISA-Internexa \\
\hline Mbps & $\begin{array}{l}\text { Ancho de banda contratado por cada } \\
\text { cliente. Se presenta en Mbps. }\end{array}$ & Mensual & ISA-Internexa \\
\hline Servicios & $\begin{array}{l}\text { Número de servicios contratados por } \\
\text { cada cliente. }\end{array}$ & Mensual & ISA-Internexa \\
\hline Renegociaciones & $\begin{array}{l}\text { Toma el valor de } 1 \text { cuando se presenta } \\
\text { una renegociación, } 0 \text { en caso contrario. }\end{array}$ & Mensual & ISA-Internexa \\
\hline PIB per cápita & $\begin{array}{l}\text { Expresada en usd a precios constan- } \\
\text { tes de } 2005 .\end{array}$ & Trimestral & $\begin{array}{l}\text { Banco de la República } \\
\text { y Dane }\end{array}$ \\
\hline Demanda Global & $\begin{array}{l}\text { Ancho de banda utilizado por Co- } \\
\text { lombia. Expresada en Gigabytes por } \\
\text { segundo. }\end{array}$ & Anual & Telegeography \\
\hline $\mathrm{IHH}$ & $\begin{array}{l}\text { Índice de Hirschman-Herfindahl } \\
\text { normalizado. }\end{array}$ & Anual & TEXTSCISA-Internexa \\
\hline Inversión en TI & $\begin{array}{l}\text { Proxi de la inversíon en tecnología. } \\
\text { Expresada en USD }\end{array}$ & Anual & $\begin{array}{l}\text { Business Monitor } \\
\text { International }\end{array}$ \\
\hline Utilidad & $\begin{array}{l}\text { Utilidad antes de impuestos. Expre- } \\
\text { sada en USD }\end{array}$ & Anual & $\begin{array}{l}\text { Superintendencia de } \\
\text { Sociedades }\end{array}$ \\
\hline
\end{tabular}

Fuente: elaboración propia a partir de Internexa (2015), Banco de la República (2015), Dane, Telegeography (2015), Business Monitor International (2015)

Como respuesta a la necesidad de un análisis más a profundidad, en el comportamiento del ancho de banda al interior del mercado, se hace necesario el estudio de los agentes involucrados en este sector, es decir, las personas responsables de la colocación de las cantidades. Este proceso, de entrada, significa un reto en el acceso a datos, ya que cada firma dificulta enormemente el acceso a estos. Como solución, se propone la utilización del índice de Herfindahl e Hirschman (IHH), normalizado ${ }^{2}$, como proxi, para el comportamiento de las

1 Vale la pena resaltar que la construcción de la variable Mbps Compañía no es más que la suma de los servicios contratados por los clientes en cada periodo de tiempo, por lo que su papel como variable explicativa del precio sera diferente, ya que se usa no agregada, como es utilizada en este escenario, sino asociada con cada cliente.

2 La estimación del IHH normalizado se debe a que la empresa de donde se obtuvieron los datos lo calcula de esta manera y, por lo tanto, no existe información para estimarlo 
firmas al interior del mercado (Rhoades, 1993), calculado como lo indica la ecuación $1^{3}$.

Tabla 4. Descripción variables

\begin{tabular}{|c|c|c|c|c|c|c|}
\hline Variable & & Media & D.E. & Min. & Max. & Observaciones \\
\hline & General & 162,07 & 108,16 & 0,134 & 475 & $\mathrm{~N}=5254$ \\
\hline \multirow[t]{3}{*}{ Tarifa } & Between & & 108,23 & 9,27 & 475 & $\mathrm{n}=183$ \\
\hline & Within & & 64,95 & $-87,11$ & 544,35 & T-bar $=28,71$ \\
\hline & General & 230,72 & 1125,571 & 1 & 35005 & $\mathrm{~N}=5254$ \\
\hline \multirow[t]{3}{*}{ Mbps } & Between & & 613,1 & 1 & 6155,05 & $\mathrm{n}=183$ \\
\hline & Within & & 893,0049 & $-5923,324$ & 29080,68 & T-bar $=28,71$ \\
\hline & General & 6,299962 & 14,18459 & 1 & 155 & $\mathrm{~N}=5254$ \\
\hline \multirow[t]{3}{*}{ Servicios } & Between & & 10,43735 & 1 & 101,1613 & $\mathrm{n}=183$ \\
\hline & Within & & 7,576285 & $-60,86133$ & 66,67496 & T-bar $=28,71$ \\
\hline & General & 41,38395 & 162,2075 & $-275,8305$ & 872,5424 & $N=1306$ \\
\hline \multirow[t]{2}{*}{ Utilidad } & Between & & 166,8393 & $-127,4842$ & 829,8642 & $\mathrm{n}=37$ \\
\hline & Within & & 53,39952 & $-316,6008$ & 288,4729 & T-bar $=35,2973$ \\
\hline PIB per cápita & & 1273,046 & 145,92 & 894,2344 & 1454,275 & 96 \\
\hline TRM & & 2053,882 & 315,3028 & 1732,286 & 3066,408 & 96 \\
\hline Demanda Global & & 699,6088 & 436,9664 & 94 & 1596 & 96 \\
\hline $\mathrm{IHH}$ & & 0,2746546 & 0,0189481 & 0,2482015 & 0,3011414 & 96 \\
\hline Inversión en TI & & 59,78498 & 16,80395 & 0,2829117 & 78,1 & 96 \\
\hline
\end{tabular}

Fuente: elaboración propia a partir de Internexa (2015); Banco de la República (2015); DANE (2015); Telegeography (2015); Business Monitor International (2015).

\section{Índice de Hirschman-Herfindahl}

$$
I H H=\frac{\frac{\sum_{i=1}^{N} s_{i}^{2}}{10000}-\frac{1}{N}}{1-\frac{1}{N}}
$$

como se realiza tradicionalmente. No es posible tener el IHH para periodos anteriores a la entrada de Azteca Comunicaciones, debido a la ausencia de datos.

3 Donde $\mathrm{S}$ es la participación porcentual de la firma en el mercado y $\mathrm{N}$ es el número total de firmas. 
Como se evidencia en la figura 3 , el mercado ha sido altamente dinámico en los últimos años, volviéndose menos concentrado con la entrada de nuevos competidores como Azteca Comunicaciones en 2008 y concentrándose de nuevo con operaciones, como la adquisición de Lazus por parte de Columbus Networks en 2012. Como estas hay muchas más operaciones a nivel local que afectan la magnitud del índice, pero lo interesante es conocer los efectos que tiene la concentración o desagregación del mercado de telecomunicaciones en el de mayoristas. La caída pronunciada del índice para el último periodo de análisis se debe a la entrada de un cable submarino (Latin American Nautilos) que conllevó al crecimiento de un mercado alterno de fibra oscura.

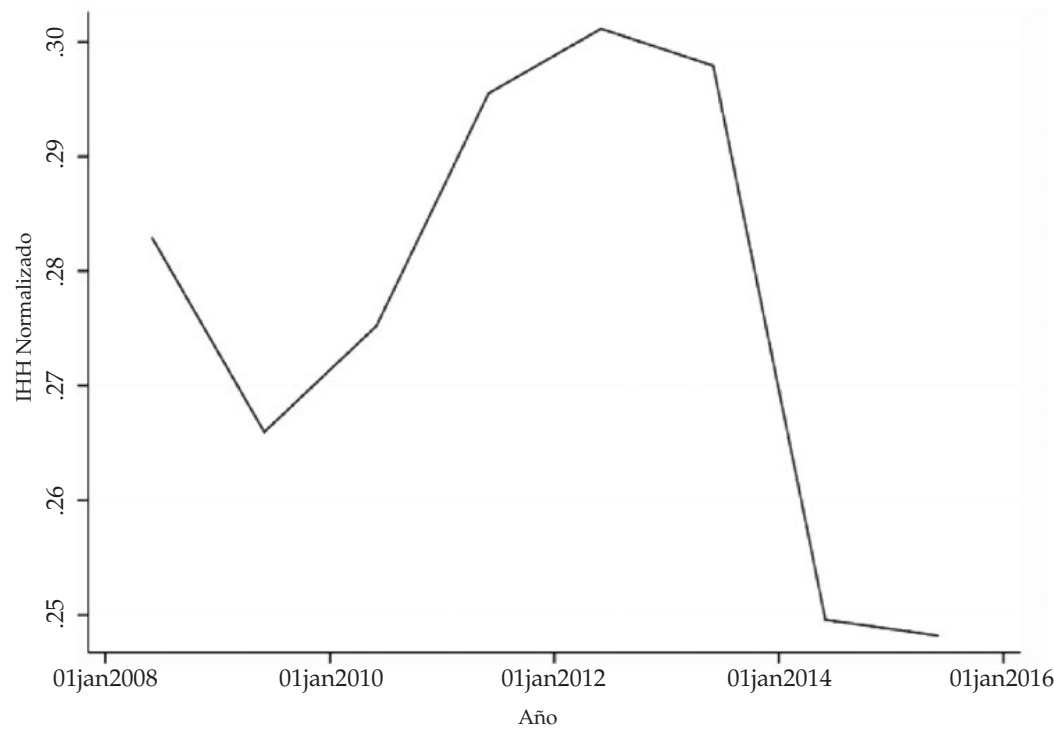

Figura 3. IHH de la industria

Fuente: elaboración propia a partir de Internexa (2015).

Desde el punto de vista teórico, los mayoristas prefieren ubicarse en mercados poco competidos, es decir, su ideal está en un mercado con IHH alto, ya que esto les permite obtener beneficios extraordinarios (Rhoades, 1993). Esta situación, característica de este tipo de mercados, se ha hecho evidente, en parte, gracias a informes como OECD (2014) y a un trabajo incipiente, en la labor de control y regulación por parte de la Comisión de Regulación en Comunicaciones (CRC).

Aunque muy importantes, la demanda del mercado y las cantidades no son los únicos determinantes externos utilizados para modelar los precios en el sector. El poder adquisitivo de los individuos y las firmas cumple un papel 
fundamental en el proceso de formación de los precios de los servicios a nivel de los mayoristas, gracias a que son estos los que actuán como usuarios finales de los servicios comercializados ya que un incremento en la demanda y en consecuencia en el precio, a este nivel, repercute en un incremento en el precio a nivel mayorista (Madden y Coble-Neal, 2004).

Para analizar el poder de compra de los consumidores, vale la pena reconsiderar la capacidad que tienen las firmas que proveen los servicios de transporte de datos, es decir, las empresas que son consumidoras de los servicios provistos por los mayoristas. Para esto, se presenta en la figura 4, una serie construida a partir de las medias de las utilidades de las empresas consumidoras de Internexa.

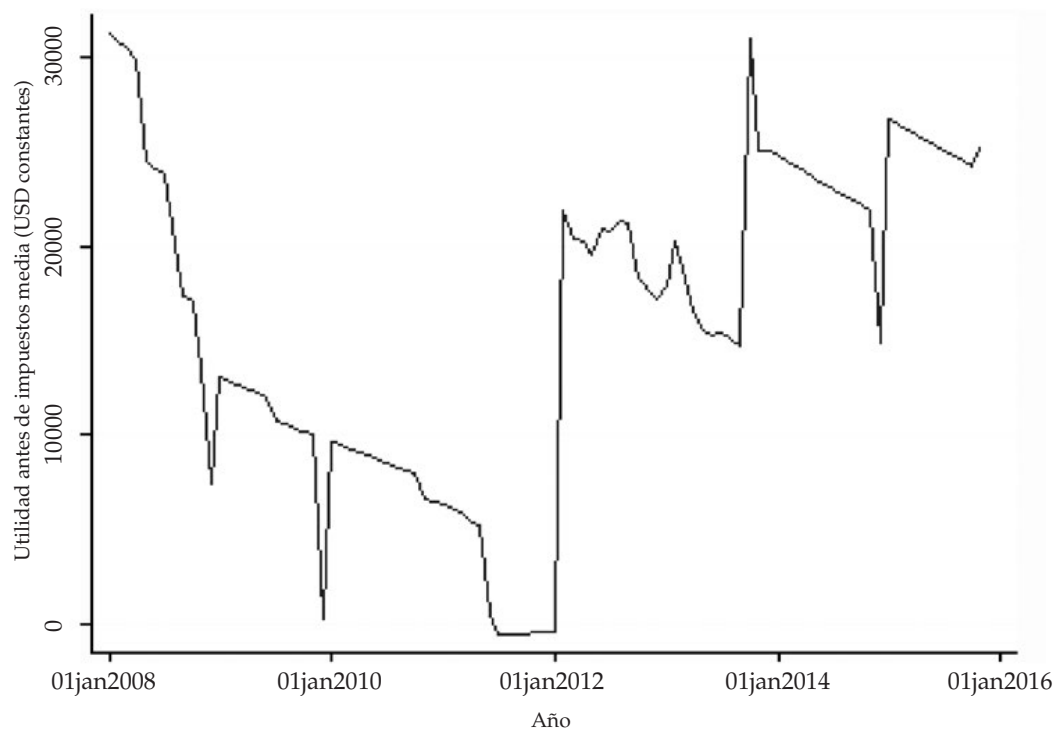

Figura 4. Utilidad media de los clientes

Fuente: elaboración propia a partir de Superintendencia de Sociedades (2015).

La figura 4 permite apreciar cómo el sector minorista de las telecomunicaciones resultó muy golpeado, entre otras cosas, por la crisis de 2008 y solo hasta el 2012 pudo recuperarse. Esta situación no es ajena, como se verá posteriormente, al sector mayorista, ya que una baja en el ingreso de sus clientes repercute en sus propios ingresos.

Desde el punto de vista de los consumidores se podría decir que teóricamente, se esperaría un efecto positivo del pib en el precio. Con el objetivo de estimar esta relación, se establece como proxi del nivel adquisitivo de los consumidores, el PIB per cápita que, hasta el primer trimestre de 2014, venía 
con un incremento constante y cayó abruptamente, debido a la importante depreciación del peso colombiano frente al dólar, fruto de la caída internacional de los precios del petróleo, lo que afectó los términos de intercambio de los colombianos.

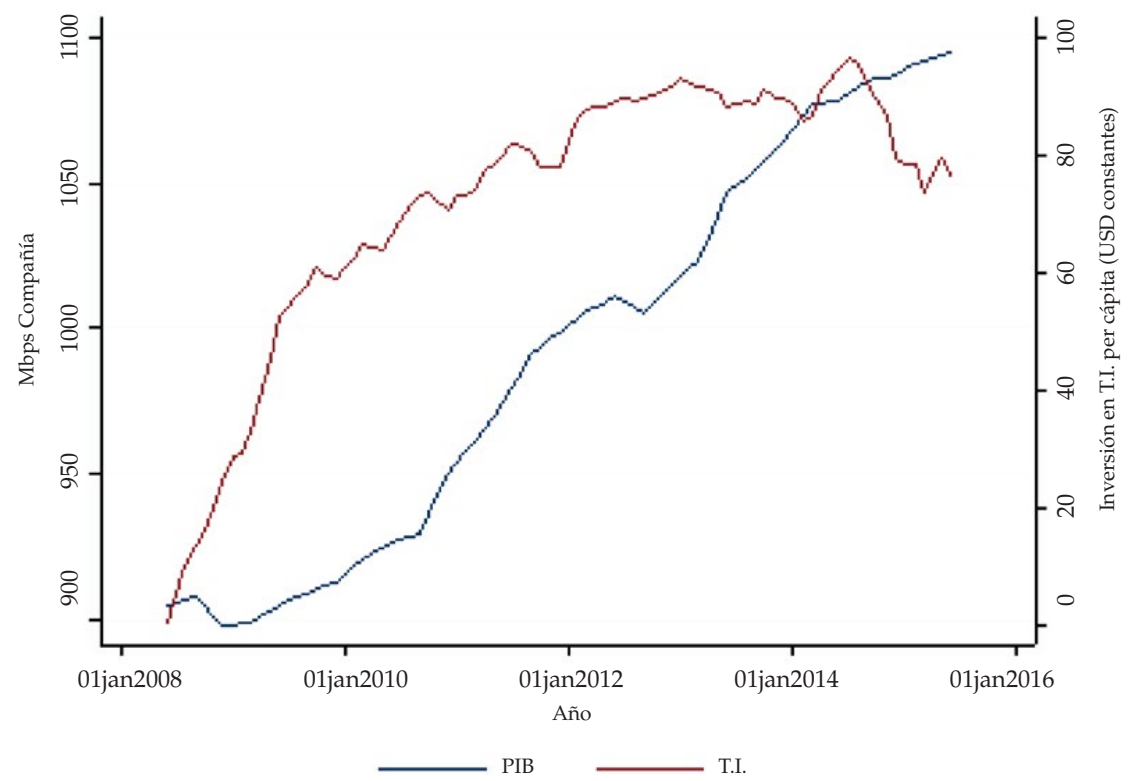

Figura 5. PIB e Inversión en TI per cápita

Fuente: elaboración propia a partir de Banco de la Republica (2015); DANE (2015); Business Monitor International (2015).

Por último, se presenta la inversión en tecnologías de información (TI) per cápita como proxi de la inversión en el sector, cumpliendo la función, no solo de controlar por la innovación tecnológica, sino también de calcular el impacto que esta tiene sobre la tarifa. Como se puede apreciar en la figura 5, la relación entre la inversión en TI y el PIB per cápita es muy estrecha, pero la diferencia radica en el comportamiento frente a la crisis internacional de los precios del petróleo (Madden y Coble-Neal, 2004).

Las telecomunicaciones, como un sector ampliamente dependiente de la tecnología, requiere de altas inversiones para permanecer en el mercado y atraer nuevos clientes (Jankowski et al., 2014; OECD, 2014), no basta solo con existir, para crecer y permanecer en la industria, se requiere de un incremento en el valor agregado diferenciando el servicio que se ofrece a los clientes para ser competitivos. 


\section{Metodología}

Se plantea la realización de dos modelos por panel de datos dinámico (Blundell y Bond), con el objetivo de encontrar los determinantes más importantes del precio y la demanda del servicio de ip en el mercado mayorista de telecomunicaciones. Dicho panel cuenta con un periodo de 8 años, con periodicidad mensual, para 96 periodos.

El planteamiento del modelo se basó en el trabajo realizado por Karacuka et al. (2011), se prefirió este trabajo por encima de autores como Madden y Coble-Neal (2004) o Hausman (1999), no porque su modelo teórico fuera mejor o más convincente, sino por el hecho de que trabajaba a nivel de firmas, al igual que este trabajo, y se encontraban soluciones a diversos problemas a los que se enfrentó esta investigación. Por supuesto, también se integraron los trabajos de Madden y Coble-Neal (2004) y Hausman (1999) para complementar y argumentar la especificación del modelo en esta investigación.

\subsection{Regresores y regresando}

Como resultado de la convergencia en la metodología de varios autores, especialmente los anteriormente mencionados, se llega al planteamiento de dos modelos, estimados por separado, ya que representan procesos distintos dentro del mercado. Por un lado, el modelo de demanda permite establecer las variables que inciden en el comportamiento y crecimiento del mercado, además de permitir la estimación de las elasticidades mencionadas, mientras que, por otra parte, el modelo de formación de precios permite obtener información sobre el proceso generador de precios al interior de la industria.

Si bien, ambos modelos comparten la idea sobre la explicación del mercado, lo hacen de manera muy diferente, lo que conlleva a que sean estimados con variables distintas, que a excepción del pib per cápita y por supuesto los precios y las cantidades, no tendrían sentido de ser incluidos en la otra estimación.

$$
\begin{gathered}
P_{i, t}=\alpha+\text { Mbps }_{i, t}+\text { PIB }_{i, t}+\text { Demanda }_{i, t}+\text { TI I }_{i, t}+\text { Serv }_{i, t}+\text { Rene }_{i, t}+\mu_{i, t} \\
\text { Mbps }_{i, t}=\alpha+P_{i, t}+\text { IHH }_{i, t}+\text { PIB }_{i, t}+\text { Zona }_{i, t}+\text { Utilidad }_{i, t}+\mu_{i, t}
\end{gathered}
$$

El primero, un modelo de formación de precios (P), ilustrado en la ecuación 2, donde el logaritmo natural de la tarifa de cada cliente, dada en Mbps por USD, hace las veces de variable dependiente, explicada por las siguientes variables: 
1. El logaritmo natural de los Mbps contratados por cada cliente en cada periodo (Mbps).

2. El logaritmo natural de PIB per cápita (PIB).

3. El logaritmo natural de la demanda total del país por servicios de banda ancha internacional (Demanda).

4. El logaritmo natural de la inversión en TI per cápita, como proxi de la inversión del sector en tecnología (TI).

5. El número de servicios por cliente en cada periodo, como variable control para los clientes con múltiples servicios (Ser).

6. Una variable dummy de renegociaciones, la cual toma el valor de 1 cuando un servicio se ha renegociado en cada periodo (Rene).

El segundo, un modelo de demanda (Mbps), descrito en la ecuación 3, donde el logaritmo de las cantidades hace las veces de variable dependiente con los siguientes regresandos:

1. El logaritmo natural del precio, asociado con cada capacidad, dado en Mbps por USD (P).

2. El logaritmo del $\mathrm{IHH}$, construido a partir de las participaciones de los diversos agentes del mercado (IHH).

3. El logaritmo del PIB, al igual que en el modelo de formación de precios, siendo este el único regresando presente en ambas regresiones (PIB).

4. Un índice de zona de prestación de servicio como variable control (comprende valores en el rango $0-100$, donde 0 es un portafolio de servicios prestado completamente en un área rural y 100 es un portafolio de servicios prestado completamente en área urbana) (Zona).

5. El logaritmo natural de la utilidad antes de impuestos de las firmas que cumplen el papel de consumidores (utilidad).

Algunas de las series presentadas anteriormente, comprendían un problema mencionado con anterioridad, estas no presentaban una periodicidad mensual. Entre las series con este problema se encontraban ${ }^{4}$ :

4 De estas, el PIB per cápita no representaba un problema de tanta relevancia, ya que las series tenían una periodicidad trimestral, pero las demás series presentaban una periodicidad anual. Para solucionar este problema se utilizaron técnicas de interpolación para obtener datos estimados de los periodos faltantes y poder proceder con la estimación del modelo. 
1. El logaritmo natural de PIB per cápita, construido a partir de las series trimestrales del PIB y la población.

2. El logaritmo natural de la demanda total del país por servicios de banda ancha internacional.

3. El logaritmo natural de la inversión en TI per cápita, como proxi de la inversión del sector en tecnología.

4. El logaritmo natural del IHH.

5. El logaritmo natural de las utilidades antes de impuestos.

\section{Resultados}

Una vez definidos los regresores y el regresando, se realizaron pruebas de multicolinealidad, haciendo uso del Factor de Inflación de Varianza para controlar por este problema. Se hallaron valores bajos del vif (ver tablas 7 y 8), tanto para el modelo de formación de precios como para el modelo de demanda, con un vif promedio de 1,52 y 1,32, por lo que podemos concluir que no existen problemas de varianza inflada que sesguen los betas (Greene, 2003; Stine, 1995).

Se procedió a realizar pruebas para la especificación del modelo, como el test de especificación de Hausman, con el objetivo de determinar la naturaleza de los efectos presentes en la estimación (Greene, 2003), dicho test arrojó una diferencia muy pequeña en los betas, lo que sugirió la presencia de efectos fijos (ver tablas 11 y 12).

Con el objetivo de controlar por la endogeneidad entre los precios y las cantidades, presente en ambos modelos, se propone la utilización de los precios y de las cantidades rezagadas, como instrumento, haciendo uso del tiempo de retraso natural en los contratos del mercado mayorista.

\subsection{Regresión principal}

Los principales resultados provenientes del modelo de demanda, son sin lugar a dudas, los $\beta$ asociados con el $\log M b p s$ y logUtilidad, ya que estos reportan las elasticidades que se pretendían estimar, dando como resultado una elasticidad precio de la demanda de $-0,27$ y una elasticidad ingreso, para las firmas proveedoras de servicios a los usuarios finales, de 0,038 (tabla 5).

Como era de esperarse, la elasticidad precio de la demanda tiene un signo negativo, conservando la ley de la demanda. Además, se trata de una elasticidad inelástica $(-0,27)$, lo cual es consistente para el periodo de análisis, con el funcionamiento de esta industria, donde a pesar de que para el 2012 se dió la 
adquisición de Lazus por Columbus Networks; también se presentó la entrada de otras empresas al sector, como Azteca Comunicaciones en 2008 y en 2014 entró en operación el cable Latin American Nautilus (LAN), lo que posibilitó el crecimiento de un mercado de fibra oscura y una mayor competencia en la industria, con altas inversiones en tecnología.

Tabla 5. Regresiones modelo demanda

\begin{tabular}{|c|c|c|c|c|}
\hline & $\begin{array}{l}\text { Efectos fijos } \\
\log \text { T arif at }\end{array}$ & $\begin{array}{l}\text { Efectos fijos } \\
\log \text { T arif at }-1\end{array}$ & $\begin{array}{l}\text { Efectos fijos } \\
\log \text { T arif at }-2\end{array}$ & $\begin{array}{c}\text { Blundell y Bond } \\
\log \text { arif at }-2 \\
\text { logUtilidadt-3 }\end{array}$ \\
\hline \multirow{2}{*}{ Constante } & 4,020751 & 2,787046 & 2,891253 & 0,7243555 \\
\hline & $(1,829926)$ & $(1,90077)$ & $(1,937354)$ & $(1,337039)$ \\
\hline \multirow{2}{*}{ logTarifa } & $-1,110248^{* * *}$ & $-1,06179^{* * *}$ & $-1,062488^{* * *}$ & $-0,2768294^{* * *}$ \\
\hline & $(0,0391154)$ & $(0,0428657)$ & $(0,0450397)$ & $(0,0403798)$ \\
\hline \multirow{2}{*}{$\log \mathrm{TI}$} & $0,7990172^{* *}$ & $0,5939182^{*}$ & $0,6234582^{*}$ & $0,75887624^{*}$ \\
\hline & $(0,3303756)$ & $(0,3409152)$ & $(0,3440681)$ & $(0,321369)$ \\
\hline \multirow{2}{*}{$\log$ PIB } & $0,8983251^{* * *}$ & $0,9978984^{* * *}$ & $0,9772288^{* * *}$ & $0,5018719^{* * *}$ \\
\hline & $(0,1932784)$ & $(0,1987288)$ & $(0,2015731)$ & $(0,1496602)$ \\
\hline \multirow{2}{*}{ Zona } & $-0,0182325^{* * *}$ & $-0,0188214^{* * *}$ & $-0,0188355^{* * *}$ & $-0,0132134^{* * *}$ \\
\hline & $(0,0012172)$ & $(0,0012515)$ & $(0,0012669)$ & $(0,001446)$ \\
\hline \multirow{2}{*}{ logUtilidad } & $0,0569096^{*}$ & $0,0613837^{*}$ & $0,0683256^{* *}$ & $0,0380288^{* *}$ \\
\hline & $(0,0223839)$ & $(0,0228901)$ & $(0,0232017)$ & $(0,0141079)$ \\
\hline$R 2$ & 0,4895 & 0,4717 & 0,4734 & 0,6845 \\
\hline R2 Between & 0,4734 & 0,4575 & 0,5277 & - \\
\hline$R 2$ Within & 0,7001 & 0,7590 & 0,7583 & - \\
\hline
\end{tabular}

* Estadísticamente significativo a un nivel del $10 \%$.

** Estadísticamente significativo a un nivel del $5 \%$.

*** Estadísticamente significativo a un nivel del $1 \%$.

Fuente: elaboración propia.

Al comparar esta elasticidad con estudios previos, como se mencionó en la revisión de literatura, como los de Karacuka et al. (2011); Grzybowski (2008); Rodini et al. (2003); Ahn y Lee (1999), se puede observar que tiende a ser una de las más bajas, pero aun se caracteriza por ser inelástica, al igual que las encontradas en estos estudios.

Respecto a la elasticidad ingreso de la demanda, también presenta un coeficiente acorde con la teoría económica, el cual permite establecer que el servicio de ip se comporta como un bien normal necesario. 
En lo correspondiente al pib per cápita, se obtiene el resultado esperado, ya que las cantidades reaccionan positivamente al incremento de este, resultado fácilmente explicable desde la teoría microeconómica como un aumento en el ingreso que deriva en un aumento del consumo.

Se presenta también, el coeficiente estimado sobre el IHH, el cual es de bastante importancia, ya que describe como reacciona el mercado frente a cambios o movimientos en su interior. Este coeficiente muestra que, a diferencia de muchos mercados industriales, el de mayoristas en telecomunicaciones colombiano reacciona de una manera positiva a la concentración del mercado, con lo que se obtienen mayores beneficios cuando este es más concentrado.

Finalmente, en relación con la zona de prestación del servicio de IP, se encuentra evidencia de un fenómeno bastante predecible en Colombia, el cual describe una marcada tendencia a que se presten mayor cantidad de servicios de internet en las zonas urbanas. El acceso a Internet en las zonas rurales aun no ha tenido un desarrollo total.

Desde la organización industrial de un sector, como lo estblecen Lipczynski, Wilson, Goddard y Goddard (2005); Grether (1970), cada vez toma mayor relevancia el paradigma estructura-conducta-desempeño, popularizado por Bain y Mason en los cincuenta, para explicar el funcionamiento de esta. Desde el desempeño, de acuerdo con Einav y Levin (2010), una variable clave a explicar, es la formación de precios, motivo por el cual se analizará el modelo de formación de pecios, el cual conserva la ley inversa de la demanda con un coeficiente de -0,0288 (tabla 6).

Respecto a la inversión en tecnología, se puede ver que esta es una forma de incrementar la tarifa, vía diferenciación del producto. Este coeficiente es de gran importancia, ya que es una forma endógena de elevar las tarifas, sin tener que acudir a la alteración de las cantidades en el mercado y, por lo tanto, un mercado donde existe tendencia a la baja de los precios.

Tabla 6. Regresiones modelo formación de precios

\begin{tabular}{lcccc}
\hline & $\begin{array}{c}\text { Efectos fijos } \\
\log M b p s t\end{array}$ & $\begin{array}{c}\text { Efectos fijos } \\
\log M b p s t-1\end{array}$ & $\begin{array}{c}\text { Efectos fijos } \\
\text { logMbpst-2 }\end{array}$ & $\begin{array}{c}\text { Blundell y Bond } \\
\log \text { ogpst-2 }\end{array}$ \\
\hline Constante & 4,524074 & 4,776372 & 4,831962 & 0,9494948 \\
& $(0,3305405)$ & $(0,3162219)$ & $(0,3141791)$ & $(0,2027256)$ \\
$\log \mathrm{Mbps}$ & $-0,2215718^{* * *}$ & $-0,2312378^{* * *}$ & $-0,237973^{* * *}$ & $-0,0288542^{* * *}$ \\
& $(0,0068666)$ & $(0,0071867)$ & $(0,0076345)$ & $(0,005744)$ \\
$\log \mathrm{PIB}$ & $0,4984017^{* * *}$ & $0,4774089^{* * *}$ & $0,4760633^{* * *}$ & $0,195205^{* * *}$ \\
& $(0,0474808)$ & $(0,0454573)$ & $(0,0451139)$ & $(0,0262918)$
\end{tabular}




\begin{tabular}{lcccc}
\hline & $\begin{array}{c}\text { Efectos fijos } \\
\text { logMbpst }\end{array}$ & $\begin{array}{c}\text { Efectos fijos } \\
\text { logMbpst }-1\end{array}$ & $\begin{array}{c}\text { Efectos fijos } \\
\text { logMbpst-2 }\end{array}$ & $\begin{array}{c}\text { Blundell y Bond } \\
\text { logMbpst-2 }\end{array}$ \\
\hline \multirow{2}{*}{$\log$ Dem } & $-0,4624514^{* * *}$ & $-0,4719925^{* * *}$ & $-0,4714661^{* * * *}$ & $-0,1400305^{* * *}$ \\
& $(0,0130364)$ & $(0,0126382)$ & $(0,0127767)$ & $(0,0113354)$ \\
$\log$ Tech & $0,0675594^{* * *}$ & $0,0685517^{* * *}$ & $0,0625347^{* * *}$ & $0,0167853^{* *}$ \\
& $(0,0112486)$ & $(0,0106557)$ & $(0,0108888)$ & $(0,0061087)$ \\
Servicios & $0,0070238^{* * *}$ & $0,0068215^{* * *}$ & $0,0067508^{* * *}$ & 0,0006551 \\
& $(0,0006165)$ & $(0,0005868)$ & $(0,000578)$ & $(0,0005242)$ \\
Rene & $-0,0817604^{*}$ & $-0,0860605^{*}$ & $-0,0865705^{*}$ & $-0,1575358^{* * *}$ \\
$R 2$ & $(0,0416299)$ & $(0,0396546)$ & $(0,0393027)$ & $(0,0191937)$ \\
$R 2$ Between & 0,6060 & 0,6281 & 0,6385 & 0,8278 \\
$R 2$ Within & 0,6328 & 0,6378 & 0,6392 & - \\
\hline
\end{tabular}

* Estadísticamente significativo a un nivel del $10 \%$.

** Estadísticamente significativo a un nivel del $5 \%$.

*** Estadísticamente significativo a un nivel del $1 \%$.

Fuente: elaboración propia.

$\mathrm{Al}$ analizar los resultados obtenidos para la cantidad de Gbps en el mercado, se obtiene un resultado acorde con lo esperado, según la teoría económica. Un $\beta=-0,14$ explica una relación inversa entre las cantidades totales en el mercado y el precio de estos servicios. Resalta la magnitud, que describe con increíble precisión la volatilidad que ha tenido el mercado en los últimos años, con caídas anuales del orden del $30 \%$.

Con respecto a los servicios, se obtiene un resultado que a primera vista podría parecer contra intuitivo, pero que se explica como una transmisión mayor de valor, al entregar una capacidad en diferentes lugares. A pesar de que con el enfoque Blundell y Bond no resulta estadísticamente significativo, un coeficiente positivo explica que cada servicio adicional que un cliente adquiera eleva su tarifa por mega, respuesta que captura un efecto que vale la pena resaltar en el mercado colombiano.

Adicionalmente, el resultado obtenido para la variable renegociaciones, muestra un reducción de poco más del $15 \%$ cuando se presenta una renegociación de un contrato. Este resultado es muy interesante pues habla, no solo de la cultura de la negociación en Colombia, la cual valdría la pena investigar a fondo, sino también de la posibilidad de prever estos cambios y generar políticas enfocadas a mitigar las caídas de precios generadas por estas negociaciones. 


\section{Conclusiones}

En conclusión, se puede ver cómo el proceso de formación de precios en el mercado de mayoristas en telecomunicaciones, está determinado, principalmente, por variables como el ancho de banda contratado, la capacidad de compra de los consumidores finales (PIB per cápita), la inversión realizada en tecnología (Inversión en TI), la cantidad total de Gigabytes en el mercado, el número de servicios contratados por un cliente y las renegociaciones que se hagan del contrato. Variables descritas, en su mayoría, por autores como Karacuka et al. (2011) y Madden y Coble-Neal (2004).

Mientras que las cantidades, por otro lado, están descritas por variables como la tarifa de los servicios, el comportamiento de la industria, medido por el IHH, el poder de compra de los consumidores, medido por el PIB per cápita, la zona de prestación del servicio y los ingresos de las compañías, medido en su utilidad antes de impuestos.

Por medio de la utilización de un modelo de demanda, estimado mediante Blundell y Bond, inspirados en el utilizado por Hausman (1999) para estimar una función de demanda, se encontró que todas las variables anteriormente descritas cumplen un papel importante en la determinación de los precios y las cantidades en un mercado altamente dinámico y poco regulado como es el colombiano.

Dichos procesos de formación, tanto de precios como de cantidades a colocar en el mercado, probaron que el mercado mayorista de telecomunicaciones colombiano, está altamente ligado al minorista, tanto así que la elasticidad precio de la demanda es comparable con algunas encontradas en estos mercados y prueba que los mercados industriales deberían ser objeto de un mayor estudio, no solo para tener un mejor entendimiento de los mercados a los que acceden los consumidores finales, sino también para poder diseñar mejores políticas públicas con el fin de tener una mejor regulación en el sector de las telecomunicaciones.

Este trabajo se enfocó en encontrar los determinantes de la demanda en un sector bastante marginado por la investigación científica, sea por la falta de interés o por el difícil acceso a los datos. Pero se espera que los hallazgos y el análisis del sector sean de utilidad no solo para futuras investigaciones del sector, sino también para los entes reguladores como la CRC, ya que se presentan herramientas cuantitativas, las cuales pueden ser de gran utilidad para la mejora de la competitividad del mercado. 


\section{Anexos}

\subsection{Pruebas de multicolinealidad}

Tabla 7. Test de multicolinealidad, modelo de formación de precios

\begin{tabular}{lcccc}
\hline & VIF & SQRT VIF & Tolerancia & R`2 \\
\hline $\log$ Mbps & 1,33 & 1,15 & 0,7547 & 0,2453 \\
$\log$ IIB & 1,25 & 1,12 & 0,8022 & 0,1978 \\
$\log$ Dem & 2,30 & 1,52 & 0,4352 & 0,5648 \\
$\log$ Tech & 1,92 & 1,39 & 0,52 & 0,4800 \\
Servicios & 1,29 & 1,14 & 0,7725 & 0,2275 \\
Rene & 1,01 & 1 & 0,99 & 0,0088 \\
\hline
\end{tabular}

Tabla 8. Test de multicolinealidad, modelo de demanda

\begin{tabular}{lcccc}
\hline & VIF & SQRT VIF & Tolerancia & R`2 \\
\hline $\log$ Tarifa & 1,26 & 1,12 & 0,7909 & 0,2091 \\
$\log$ IHH & 1,49 & 1,22 & 0,6697 & 0,3303 \\
$\log$ PIB & 1,59 & 1,26 & 0,6299 & 0,3701 \\
Zona & 1,16 & 1,07 & 0,8658 & 0,1342 \\
$\log$ Utilidad & 1,09 & 1,04 & 0,9213 & 0,0787 \\
\hline
\end{tabular}

\subsection{Pruebas de especificación}

Tabla 9. Test deBreusch-Pagan, modelo de formación de precios

\begin{tabular}{lcc}
\hline \multicolumn{1}{r}{ Test de Breusch-Pagan } & Var & SE \\
\hline logTarifa & 0,5968603 & 0,7725673 \\
Cliente & 0,1032434 & 0,3213152 \\
Cliente, tiempo & 0,1887113 & 0,4344091 \\
\hline
\end{tabular}

$$
P>\chi 2=0,0000
$$


Tabla 10. Test de Breusch-Pagan, modelo de demanda

\begin{tabular}{lcc}
\hline \multicolumn{1}{c}{ Test de Breusch-Pagan } & Var & SE \\
\hline logMbps & 4,795587 & 2,189883 \\
Cliente & 0,2077562 & 0,4558028 \\
Cliente, tiempo & 1,205961 & 1,098163 \\
\hline
\end{tabular}

$$
P>\chi 2=0,0000
$$

Tabla 11. Test de Hausman, modelo de formación de precios

\begin{tabular}{lcc}
\hline \multicolumn{1}{c}{ Variable } & Diferencia & SE \\
\hline $\operatorname{logMbps}$ & $-0,0014064$ & 0,0026072 \\
$\log \mathrm{PIB}$ & $-0,0065838$ & 0,0050946 \\
$\log \mathrm{Dem}$ & 0,0043044 & 0,0032751 \\
$\log$ Tech & $-0,0006881$ & 0,0015057 \\
Servicios & $-0,0000264$ & 0,0001105 \\
Rene & $-0,0008573$ & 0,0016582 \\
\hline
\end{tabular}

$$
P>\chi 2=0,6562
$$

Tabla 12. Test de Hausman, modelo de demanda

\begin{tabular}{lcc}
\hline \multicolumn{1}{c}{ Variable } & Diferencia & SE \\
\hline $\log$ Tarifa & 0,0169055 & 0,0026072 \\
$\log \mathrm{IHH}$ & $-0,1127621$ & 0,0050946 \\
$\log$ PIB & 0,0556802 & 0,0032751 \\
Zona & $-0,000363$ & 0,0015057 \\
$\log$ Utilidad & 0,0121413 & 0,0001105 \\
\hline
\end{tabular}

$$
P>\chi 2=0,6562
$$

\section{Referencias}

Ahn, H. y Lee, M. H. (1999). An econometric analysis of the demand for access to mobile telephone networks. Information Economics and Policy, 11 (3), 297-305. 
Azteca-Comunicaciones (1999). Red nacional de fibra óptica. Recuperado de http:/ / aztecacomunicaciones.com

Coletti, P. (2010). Advanced Statistics. Free University of Bolzano Bozen.

Cusano, J. (2014). The connected insurer-part two: How can carriers benefit from the internet of things? Transcripción de entrevista radial. Recuperado de http:/ /insuranceblog.accenture.com/the-connected-insurerhow-can-carriers-benefit-2-of-2

Dewenter, R. y Haucap, J. (2008). Demand elasticities for mobile telecommunications in austria. Jahrbu"cher fu"r Nationalo"konomie und Statistik, 228 (1), 49-63.

Einav, L. y Levin, J. D. (2010). Empirical industrial organization: A progress report. Journal of Economic Perspectives, 24 (2), 145-162.

Greene, W. H. (2003). Econometric Analysis (5. ed.). Upper Saddle River, NJ: Prentice Hall.

Grether, E. T. (1970). Industrial organization: past history and future problems. The American Economic Review, 60 (2), 83-89.

Gruber, H. (1999). An investment view of mobile telecommunications in the european union. Telecommunications Policy, 23 (7), 521-538.

Grzybowski, L. (2008). The competitiveness of mobile telephony across the european union. International Journal of the Economics of Business, 15 (1), 99-115.

Hausman, J. (1999). Cellular telephone, new products, and the cpi. Journal of business $\mathcal{E}$ economic statistics, 17 (2), 188-194.

Houthakker, H. y Taylor, L. (1970). Consumer demand in the United States: analyses and projections. Harvard economic studies. Harvard University Press.

Internexa (2012). International internet bandwith by country 2003-2012. Reporte técnico, Internexa.

Internexa (2014). Analisis competitivo operacion colombia. Reporte técnico, Internexa.

Jankowski, S., Covello, J., Ritchie, J., y Costa, D. (2014). The internet of things: Making sence of the next mega-trend. Reporte técnico, Goldman Sachs.

Karacuka, M., Haucap, J., y Heimeshoff, U. (2011). Competition in turkish mobile telecommunications markets: Price elasticities and network substitution. Telecommunications Policy, 35 (2), 202-210.

Lipczynski, J., Wilson, J., Goddard, J., y Goddard, J. (2005). Industrial organization: competition, strategy, policy. Pearson Education. Prentice Hall/ Financial Times.

Madden, G. y Coble-Neal, G. (2004). Economic determinants of global mobile telephony growth. Information Economics and Policy, 16 (4), 519-534. 
Muñoz, T. G. (1996). Demand for national telephone traffic in spain from 1985-1989: An econometric study using provincial panel data. Information Economics and Policy, 8 (1), 51-73.

Muñoz, T. G. y Amaral, T. P. (1996). Demand for international telephone traffic in spain: An econometric study using provincial panel data. Information Economics and Policy, 8 (4), 289-315.

Parker, P. M. y R“oller, Hendrik, L. (1997). Collusive conduct in duopolies: multimarket contact and cross-ownership in the mobile telephone industry. The RAND Journal of Economics, 304-322.

Philipp G., G. C. y Steinhaüser, S. (2015). The double game of digital strategy. Reporte técnico, Boston Consulting Group.

Rhoades, S. A. (1993). Herfindahl-hirschman index, the. Fed. Res. Bull., 79, 188.

Rodini, M., Ward, M. R., y Woroch, G. A. (2003). Going mobile: substituta- bility between fixed and mobile access. Telecommunications Policy, 27 (5), 457-476.

Schaller, R. R. (1997). Moore's law: past, present and future. Spectrum, IEEE, 34 (6), 52-59.

Stine, R. A. (1995). Graphical interpretation of variance inflation factors. The American Statistician, 49 (1), 53-56.

Telegeography (2015). Global bandwith forecast. Reporte técnico.

OECD (2014). Estudio de la ocde sobre políticas y regulación de telecomu- nicaciones en colombia.

PWC (2015). Lessons from gigital leaders 10 attributes driving stronger performance. Reporte técnico, PWC.

Tishler, A., Ventura, R., y Watters, J. (2001). Cellular telephones in the israeli market: the demand, the choice of provider and potential revenues. Applied Economics, 33 (11), 1479-1492.

UK Competition Commission (2003). Vodafone, o2, orange and t-mobile: Reports on references under section 13 of the telecommunications act 1984 on the charges made by vodafone, $\mathrm{o} 2$, orange and t-mobile for terminating calls from fixed and mobile networks. Reporte técnico. 
